\section{Infecção por HPV em homens: Importância na transmissão, tratamento e prevenção do vírus}

\author{
HPV infection in men: Importance in transmission, \\ treatment and prevention of the virus
}

Iris Mattos Santos ${ }^{[a]}$, Mariana Franzoni Maioral ${ }^{[a]}$, Patrícia Haas ${ }^{[\mathrm{b}]}$

\section{Resumo}

0 papilomavírus humano (HPV) está diretamente relacionado ao desenvolvimento do câncer de colo de útero, e é também um importante fator de risco para outros tipos de câncer como o de vagina, vulva, pênis, ânus e nasofaringe. Além das mulheres, é sabido que o HPV também é capaz de infectar os homens, sendo que nestes, o vírus pode provocar verrugas anogenitais, papilomatoses, condilomas nasofaríngeos e também alguns tipos de neoplasias. Além disso, os homens são uma importante fonte de transmissão e propagação dessa doença sexualmente transmissivel. Nos últimos anos, muitos estudos vêm sendo realizados com o objetivo de desenvolver vacinas capazes de combater a propagação do vírus entre mulheres e, consequentemente, diminuir a incidência e mortalidade do câncer cervical. Apesar deste conhecimento, poucos estudos são voltados à questão masculina e ainda não existe um consenso quanto ao desenvolvimento de possíveis esquemas de tratamento e prevenção nesses indivíduos.

Palavras-chave: Câncer. Homem. HPV. Vacina.

\begin{abstract}
Human papillomavirus (HPV) is directly related to the development of cervical cancer, and it is also an important risk factor for other cancers such as vagina, vulva, penis, anus and nasopharynx. Besides women, it is known that HPV can also infect men, bearing in mind that in men the virus may cause anogenital warts, papillomatosis, nasopharyngeal warts and some types of cancer. Furthermore, men are important sources of transmission of this sexually transmitted disease to their partners. In recent years, many studies have been conducted with the goal of developing vaccines capable of combating the spread of HIV among women and to reduce the incidence and mortality by cervical cancer. Despite this knowledge, few studies have focus on male subjects and there is still no consensus about the development of possible treatment and prevention regimens towards them.
\end{abstract}

Keywords: Cancer. HPV. Men. Vaccine.

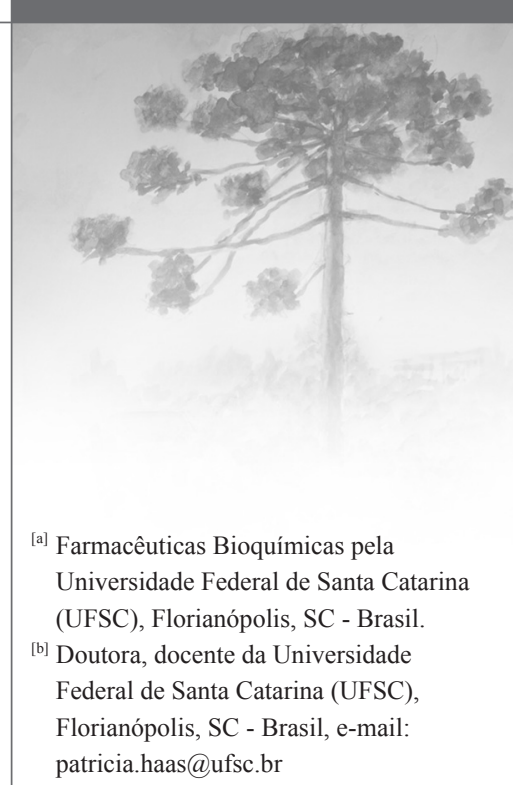

Recebido: 13/03/2011

Received: 03/13/2011

Aprovado: 13/12/2011

Approved: $12 / 13 / 2011$ 


\section{Argumentações}

Aproximadamente 15\% de todos os casos de câncer estão associados a agentes infecciosos (1), dentre eles o papilomavírus humano (HPV). Este vírus da família Papovaviridae apresenta mais de 100 tipos reconhecidos $(2,3)$ e é o agente causador da mais comum infecção sexualmente transmissível do mundo (4). De acordo com o potencial oncogênico, os tipos de HPV são divididos em três grupos, ou seja, vírus de baixo, intermediário e alto risco (5-8). Além do câncer cervical, de ânus, vulva e pênis, o vírus tem sido associado a outros cânceres epiteliais envolvendo a pele, a laringe e o esôfago, e está também estabelecida a relação entre HPV e câncer de cabeça e pescoço (9). Apesar de este vírus ser capaz de acometer homens e mulheres, ainda existem poucas informações a respeito da infecção em indivíduos do sexo masculino (10). Estudos indicam que o homem pode ser um importante agente transmissor e propagador do vírus, contribuindo indiretamente para o alto número de neoplasias cervicais. Além disso, tal como acontece com as mulheres, os homens podem experimentar significativa morbidade e potencial mortalidade nas doenças relacionadas ao HPV (6).

\section{Fisiopatologia}

O HPV é um vírus que afeta exclusivamente as células epiteliais e sua replicação está relacionada ao processo de diferenciação dessas células no hospedeiro (6). 0 vírus do HPV penetra na célula por meio da membrana basal, provavelmente pela formação de microporos, e inicia sua replicação no núcleo da célula infectada, enquanto que a produção de vírus maduros ocorre na membrana suprabasal. 0 genoma viral, mantido nessas células sob a forma de plasmídeo $(1,6)$, apresenta uma região regulatória (LCR), uma região envolvida na resistência e replicação viral, a qual é expressa precocemente (E), e outra que se expressa tardiamente (L) formadora do capsídeo viral (11). Entre as proteínas da região E, somente E6 e E7 são constitutivamente expressas em uma progressão maligna, sendo essenciais para a manutenção do fenótipo alterado e para o ciclo de vida viral (12). A expressão desses genes instituem em fibroblastos e queratinócitos, características de células imortais graças à capacidade do vírus de interação com algumas proteínas celulares. Estudos demonstram que a proteína codificada pela região E6 é capaz de se ligar e promover a degradação da p53 (fator de supressão tumoral) e outros fatores celulares como a telomerase que são responsáveis pela transdução de sinais, estímulo à apoptose e regulação da transcrição. Já a oncoproteína E7 age degradando a proteína do retinoblastoma e interfere em fatores que regulam a proliferação celular (especialmente no período compreendido entre as fases G1 e S - período de síntese e duplicação de DNA) conferindo à célula características de imortalidade $(11,13,14)$.

\section{Epidemiologia}

O HPV é responsável pela doença sexualmente transmissível mais comum do planeta e estima-se que pelo menos $75 \%$ da população sexualmente ativa já tenha sido exposta ao vírus (15), sendo que as maiores prevalências são encontradas na África, América Central e México e as menores, na Europa e Ásia $(16,17)$. 0 Brasil, bem como os países integrantes do Mercosul, não apresenta dados estatísticos significativos de prevalência de infecção pelo HPV na população sexualmente ativa e os dados acerca da ocorrência do vírus e de seus tipos mais frequentes ainda são obtidos da análise de pacientes portadoras de neoplasias intraepiteliais cervicais e de carcinoma invasivo do colo uterino (18). Assim, o Brasil pode ser considerado um país com alta incidência de infecções pelo HPV, e o Ministério da Saúde registra a cada ano aproximadamente 137 mil novos casos (19). Diferentes estudos epidemiológicos indicam que os tipos de HPV prevalentes são diferentes de acordo com a região de origem, sendo que, no Brasil, o HPV 16 foi o tipo oncogênico mais encontrado (19).

Acredita-se que a infecção masculina contribua significativamente para a infecção e subsequente doença cervical em mulheres e estima-se que mais de $70 \%$ de parceiros de mulheres com infecção cervical por HPV são portadores do DNA desse vírus $(20,21)$. A infecção pelo HPV fora da região genital foi detectada em até $73 \%$ de homens saudáveis, sendo que a persistência desta infecção é menor do que nas mulheres, e a idade parece não influenciar na incidência e duração (22). Assim como ocorre nas mulheres, os tipos mais isolados nos homens relacionam-se com a forma clínica apresentada (23). 
Transmissão

0 vírus é transmitido por meio do contato com a pele e mucosas de indivíduos infectados, sendo a principal via de transmissão a partir do contato sexual. No entanto, sabe-se que o HPV pode ser adquirido por vias não sexuais, como a transmissão perinatal $(18,24)$. Foi demonstrado que vírus de baixa oncogenicidade como os tipos de 1 a 4 podem ser transmitidos por autoinoculação para a área anogenital, sem a necessidade de ato sexual. Há também descrição a respeito da existência do vírus em espéculos vaginais, pinças e ponta de sondas, mesmo após a esterilização (20). O HPV está também relacionado ao câncer de cabeça e pescoço, e os mecanismos de transmissão nessa situação incluem transmissão perinatal, autoinfecção por contato genital-oral e transmissão a partir da prática de sexo oral (25).

\section{Clínica e diagnóstico}

A maior parte das infecções por HPV em homens apresenta caráter benigno, geralmente de forma subclínica. Pode manifestar-se como pápulas geralmente múltiplas ou como lesões carnudas e semelhantes à "couveflor" localizadas no pênis, glande, escroto, sulco balanoprepucial, região perianal e, mais raramente, no meato uretral. Podem ocorrer também lesões na cavidade oral $(23,26,27)$.

Um diagnóstico correto requer uma anamnese apropriada que indique o histórico do paciente, incluindo informações sobre o número de parceiros sexuais, os tipos de práticas sexuais e doenças sexualmente transmissíveis anteriores. Deve-se realizar também um exame físico e, se necessário, exames complementares para a pesquisa direta do vírus (28). A inspeção com ácido acético é utilizada para auxiliar na detecção da infecção, além de ser útil na triagem $(23,26)$. Pode-se utilizar uma técnica chamada colposcopia que consiste na utilização de um aparelho capaz de aumentar o poder de visão do clínico (28). Na infecção pelo HPV, o exame histológico pode apresentar acantose, que é caracterizada pelo efeito citopático do vírus nas células da epiderme (29). Existem ainda várias metodologias para detectar o genoma do HPV nas células do hospedeiro como a imunoflorescência direta, captura híbrida, a reação em cadeia da polimerase (PCR) e a hibridização in situ (26).
Neoplasias relacionadas ao HPV

0 câncer de pênis contribui com menos de 0,5\% dos casos de cânceres em homens (22). O DNA do HPV foi encontrado entre $40 \%$ e $50 \%$ dos casos de câncer de pênis, sendo que o tipo 16 foi identificado em $60 \%$ dos casos (30). 0 câncer de ânus também é uma doença rara na população em geral, mas sua incidência vem crescendo nas últimas cinco décadas, tanto em homens como em mulheres e pode ser maior se considerarmos alguns grupos de risco como indivíduos imunossuprimidos $(22,31)$. 0 DNA do HPV foi detectado em mais de $80 \%$ das lesões, e o HPV 16 foi o mais comumente isolado desses tumores, seguido pelo HPV 18 (31). A região da cabeça e pescoço compreende a cavidade oral, a orofaringe, a hipofaringe e a laringe. 0 câncer associado a essa região é muito comum, estando relacionado ao tabagismo e ao etilismo, geralmente ocorrendo entre os 60 e 70 anos de idade e sendo mais comum em homens do que em mulheres $(22,32)$. De acordo com o IARC (International Agency for Research on Cancer), 25\% dos cânceres de orofaringe relacionam-se ao HPV e estudos mostram evidências de que esse tipo de infecção esteja relacionado ao comportamento sexual do indivíduo, incluindo a prática de sexo oral. Assim como em outros cânceres, os tipos de HPV mais comumente encontrado em lesões de cabeça e pescoço foram os tipos 16 e 18 (22).

\section{Tratamento}

Dentre os métodos não invasivos disponíveis atualmente, existe uma toxina com propriedades citotóxicas chamada podofilina (33) e o ácido tricloroacético, um agente cáustico que combate verrugas anogenitais por meio do contato (34). É descrito também o uso de interferons, proteínas endógenas intracelulares que apresentam atividade antitumoral e antiviral (35) e imiquimod, um medicamento usado no tratamento de verrugas anogenitais, cujo mecanismo de ação consiste na potencialização da produção de interferons, com efeito antiviral, antiproliferativo e antiangiogênico $(36,37)$. Além destes, cidofovir é um importante antiviral usado em tumores induzidos pelo HPV, com a capacidade de provocar morte celular programada entre as células tumorais (34).

Um dos métodos invasivos mais confiáveis para a remoção de lesões únicas ou múltiplas na região 
anogenital é o método cirúrgico (33). 0 tratamento com laser apresenta eficácia entre $60 \%$ e $77 \%$, no entanto é um método de custo elevado e que pode liberar DNA viral na vaporização das lesões durante o procedimento (35). A criocirurgia é outra medida utilizada em verrugas genitais, levando à necrose da epiderme e, ocasionalmente, da parte superior da derme. É um método fácil, acessível e sem complicações graves, porém a recorrência das verrugas, segundo alguns autores, é de até $75 \%$ dos casos (34).

\section{Prevenção}

Diagnóstico e tratamento precoce: 0 rastreamento objetivando um diagnóstico precoce tem se mostrado altamente eficaz como adjuvante na prevenção de novas. Graças à baixa sensibilidade e reprodutibilidade dos métodos de triagem, as estratégias estão mudando de um modelo de prevenção baseado na detecção de lesões para a identificação biomolecular do vírus. A lógica é usar um teste mais sensível para detectar precocemente infecções de alto risco antes de atingirem a malignidade (38).

Vacinação: Atualmente estão disponíveis duas vacinas, a quadrivalente Gardasil ${ }^{\circledR}$ (tipos 6, 11, 16 e 18) e a bivalente Cervarix ${ }^{\circledR}$ (tipos 16 e 18), ambas recomendadas pelo Food and Drug Administration (FDA) para vacinação de meninas entre 11 e 15 anos de idade e para mulheres entre 16 e 26 anos de idade que não tenham sido previamente vacinadas (4). A vacinação em homens ainda é discutida e um dos seus objetivos seria uma maior redução da incidência de câncer cervical e seus precursores, uma vez que reduziria as chances de um homem infectado transmitir o vírus (39). As vacinas contra o HPV são preparadas a partir de partículas semelhantes aos vírus (VLP), produzidas por tecnologia recombinante e são administradas na forma de três injeções intramusculares de $0,5 \mathrm{~mL}$ ao longo de um período de seis meses (40). Ensaios clínicos demonstraram que as vacinas são altamente imunogênicas, seguras e bem toleradas e bastante eficazes na prevenção de infecções incidentes e persistentes, incluindo o desenvolvimento de lesões pré-malignas (41). A resposta imune à infecção genital pelo HPV é caracterizada principalmente pela imunidade celular local, a qual está associada à regressão das lesões e, possivelmente, à proteção contra uma futura infecção causada por um vírus de mesmo tipo. A imunidade humoral na forma de anticorpos neutralizantes é gerada na maioria (mas não na totalidade) dos indivíduos infectados e é dirigida contra o epítopo conformacional que a proteína de capsídio L1 exibe na superfície externa do vírus (18). Por outro lado, apesar de as atuais vacinas serem eficazes na prevenção de lesões pré-neoplásicas causadas pelo HPV 16 e 18 e até mesmo induzir imunidade celular à L1, estudos clínicos indicam que elas não têm efeito terapêutico. Isto significa dizer que indivíduos já infectados com tipos oncogênicos ainda estão em risco de desenvolver câncer. Como foi visto anteriormente, o vírus do HPV apresenta as proteínas estruturais E6 e E7 que, ao contrário dos antígenos do capsídeo, são constitutivamente expressas na progressão maligna. Assim, essas duas proteínas são alvos de escolha na tentativa de desenvolver vacinas terapêuticas voltadas para a indução da resposta imune celular contra antígenos do HPV (12). As respostas imunes envolvidas na erradicação de células tumorais são essencialmente mediadas por células $T$ CD8 + e o bloqueio das proteínas E6 e E7 reativa a proteína p53, causando apoptose da célula alterada pelo vírus (13).

Preservativos: Apesar de evidências demonstrarem que o uso de preservativos reduz substancialmente o risco de transmissão do vírus da imunodeficiência humana (HIV), estudos a respeito da efetividade dos preservativos na redução de outras infecções sexualmente transmissíveis são mais limitados (42). Um estudo realizado com 242 mulheres na Universidade de Washington sugere que os preservativos masculinos reduzem efetivamente o risco de transmissão do HPV genital, já que as mulheres cujos parceiros usavam preservativos em todas as relações sexuais durante os últimos oito meses, apresentaram uma probabilidade 70\% menor de adquirir a infecção em comparação com aquelas cujos parceiros não utilizavam preservativo (43). Já uma revisão bibliográfica realizada em 2008 demonstrou que a proteção masculina conferida pelo uso de preservativos em relações heterossexuais, pode variar de $60 \%$ a $95 \%$ (42).

Circuncisão: A circuncisão masculina é definida como a remoção cirúrgica de todo ou parte do prepúcio do pênis. Desde a década de 1980, estudos têm sugerido um efeito protetor da circuncisão na aquisição de doenças sexualmente transmissíveis, incluindo o HPV $(44,45)$. Os mecanismos biológicos envolvidos podem estar relacionados a fatores anatômicos, celulares ou ambos. A retração do prepúcio sobre o eixo durante a relação sexual expõe a mucosa interna 
do prepúcio a fluidos vaginais e cervicais, além de resultar em microlesões durante a relação sexual (46). Além disso, a cavidade úmida subprepucial pode fornecer um ambiente favorável para a sobrevivência do vírus e consequente infecção do epitélio. 0 vírus se replica em células epiteliais da epiderme e da derme e, como a mucosa interna do prepúcio é levemente queratinizada, pode facilitar o acesso do vírus às células epiteliais subjacentes em pacientes não circuncisados. Um estudo realizado com 5.534 pacientes em Uganda mostrou que a circuncisão reduziu significativamente a prevalência de infecções pelo HPV com eficácia de 35\% (45).

\section{Considerações finais}

Nas últimas décadas, foram desenvolvidos estudos a respeito da epidemiologia e patogênese de infecções genitais pelo HPV em mulheres, mas ainda existem poucas informações relacionando o vírus aos homens. Além disso, apesar de o Brasil apresentar alta prevalência dessa infecção, ainda não existem dados epidemiológicos disponíveis relacionados à população em geral, uma vez que os estudos baseiam-se principalmente em pesquisas com mulheres que apresentam exames citológicos anormais, sem considerar os indivíduos em situação subclínica. É importante ressaltar a necessidade desses tipos de estudos, pois se sabe que a prevalência e os tipos mais frequentes de HPV podem variar de acordo com a região de origem. Além disso, por se tratar de uma doença sexualmente transmissível frequente, é necessário fazer um diagnóstico correto a partir da clínica e de exames complementares, levando em consideração os indivíduos assintomáticos. Assim, é possível realizar o tratamento e o acompanhamento desse paciente a fim de reduzir os índices de transmissão e o desenvolvimento de doenças relacionadas, como neoplasias, incluindo aquelas fora da região genital.

Atualmente estão disponíveis duas vacinas: a bivalente e a quadrivalente, desenvolvidas a partir dos tipos mais frequentes no mundo. No entanto, é importante considerar a necessidade da aplicação de métodos de detecção viral para realizar sua identificação e diferenciação, pois o conhecimento a respeito da distribuição dos tipos de HPV em cada região é um fator relevante para o desenvolvimento de esquemas de vacinação. Apesar de estudos indicarem que o tipo
16 é o mais comum no Brasil, é necessário o desenvolvimento de estudos epidemiológicos em toda a população com o intuito de conhecer a prevalência dos tipos de HPV em cada área e a variação regional. Uma das questões mais importantes a considerar é se indivíduos do sexo masculino devem ser incluídos em programas de vacinação contra o HPV, já que os mesmos também podem ser infectados pelo vírus e potencialmente desenvolver doenças relacionadas, além de lesões pré-neoplásicas e neoplásicas. Apesar da pouca informação disponível, a vacina contra o HPV é autorizada e recomendada para meninos em diversos países, incluindo, por exemplo, México e Austrália. Por se tratar de uma infecção sexualmente transmissível, estudos indicam que essa inclusão poderia gerar benefícios diretos para os próprios indivíduos e também para suas futuras parceiras sexuais a partir da redução da transmissão do vírus. Além disso, um programa de vacinação eficiente poderia reduzir gastos provenientes de doenças associadas. Por outro lado, segundo Leon, 2008, a vacina contra o HPV apresenta um valor econômico elevado, custando cerca de US $\$ 450$ para as três doses necessárias. Para avaliar o valor da adição de garotos a esse programa, os benefícios à saúde e os custos econômicos devem ser comparados com aqueles associados à vacinação de meninas isoladamente. Métodos usados para medir o ganho para a saúde, calculado como o número de anos de vida salvos ajustados para a qualidade de vida durante esse período, indica que a relação custo-benefício da vacinação de 12 meninas foi calculada em US\$ 14.583 , com uma redução do câncer de colo de útero em 95\%. A inclusão de meninos no programa de vacinação reduziu em mais $5 \%$ os casos de câncer de colo de útero, tendo um custo de mais US\$ 442.039. Internacionalmente, é considerado um programa com custo-benefício satisfatório aquele de aproximadamente US\$ $50.000 \mathrm{ou}$ menos por ano de vida salvo. Sendo assim, a vacinação masculina não compensaria o investimento aplicado. No entanto, esses modelos não contemplam a redução de custos potencias necessários para o tratamento de verrugas genitais e outras lesões causadas pelo HPV, além de procedimentos cirúrgicos utilizados nos casos de papilomatose respiratória causada pela infecção vertical. Dessa maneira, a decisão final continua sendo discutida, pois ainda existe a preocupação de que a vacina contra o HPV tenha eficácia limitada para o sexo masculino.

Diferentemente do que ocorre nas mulheres com o rastreamento do câncer de colo de útero por meio do 
exame citopatológico, não existem testes disponíveis para triagem em homens, e os métodos disponíveis para a detecção do vírus são baseados em metodologias moleculares. A utilidade clínica os exames é questionável por tratar-se de uma infecção corriqueira que necessita de testes de alto custo. Além disso, o achado do DNA do vírus não indica, necessariamente, um risco elevado do desenvolvimento de uma doença grave e nenhuma terapia existente visa a erradicar a infecção assintomática. Por outro lado, é importante avaliar se esse rastreamento poderia ser relevante para a prevenção do câncer de colo de útero em mulheres.

Dilema semelhante ocorre na implementação da vacinação nos homens. Uma vez que as infecções pelo HPV podem resultar em verrugas genitais e lesões pré-neoplásicas e neoplásicas em indivíduos de ambos os sexos, deve-se considerar o custo-benefício da inclusão de homens no programa de vacinação nacional. A vacinação masculina deve ser considerada não apenas pela possibilidade de transmissão para as mulheres, mas também pelas evidências do desenvolvimento de doenças graves relacionadas ao HPV nos próprios indivíduos. Como as vacinas disponíveis atualmente têm caráter profilático, é necessária a adoção de políticas de saúde pública para alertar a população, evitando assim o primeiro contágio, seja por meio da prática de sexo seguro, da higienização de banheiros públicos e de medidas preventivas como a criação de programas de circuncisão neonatais. Além disso, é importante desenvolver uma maior conscientização da população quanto à importância da procura por serviços de urologia.

Assim, é possível sugerir que a prevenção precoce, o controle clínico regular, a informação do paciente a respeito do risco de transmissão do vírus por meio de relações sexuais e a vacinação oportuna provavelmente levarão a uma redução drástica dos casos de lesões associadas ao HPV.

\section{Referências}

1. Lehoux M, Dábramo CM, Archambault J. Molecular mechanisms of human papillomavirus-induced carcinogenesis. Public Health Genomics. 2009;12(5-6):268-80.

2. Madkan VK, Cook-Norris RH, Steadman MC, Arora A, Mendoza N, Trying SK. The oncogenic potential of human papillomaviruses: a review on the role of host genetics and environmental cofactors. Br J Dermatol. 2007;157(3):634-5.
3. Satyaprakash AK, Tyring SK. Human papillomaviruses vaccine: a dermatologic perspective. Indian J Dermatol Venereol Leprol. 2010;76(1):14-9.

4. Lepique AN, Rabachini T, Villa LL. HPV vaccination: the beginning of the end of cervical cancer? - A Review. Mem. Inst. Oswaldo Cruz. 2009;4(1):1-10.

5. Panjikovic M, Ivkovic-Kapiel T. Etiology and pathogenesis of precancerous lesions and invasive cervical carcinoma. Med Pregl. 2008;61(7-8):364-8.

6. Feller LL, Khammissa RAG, Wood NH, Lemmer J. Epithelial maturation and molecular biology of oral HPV. Infect Agent Cancer. 2009;25(4):16.

7. Parkin DM. The global health burden of infection-associated cancers in the year 2002. Int J Can Prev. 2006;118(12):3030-44.

8. Rezazadeh A, Laber DA, Ghim SJ, Jenson AB, Kloecher $\mathrm{G}$. The role of human papilloma virus in lung cancer: $\mathrm{a}$ review of the evidence. Clin Lab Sci. 2009;338(1):64.

9. Al-Daraji WI, Smith JHF. Infection and cervical neoplasia: facts and fiction. Int J Clin Exp Pathol. 2009;2(1):48-64.

10. Nielson CM, Harris RB, Flores R, Abrahamsen M, Papenfuss MR, Dunne EF, et al. Multiple-type human papillomavirus infection in male anogenital sites: prevalence and associated factors. Cancer Epidemiol Biomarkers Prev. 2009;18(4):1077-83.

11. Boccardo E, Villa LL. Viral origins of human cancer. Curr Med Chem. 2007;14(24):2526-39.

12. Sima N, Wang W, Kong D, Deng D, Xu Q, Zhou J, et al. RNA interference against HPV16 E7 oncogene leads to viral E6 and E7 suppression in cervical cancer cells and apoptosis via upregulation of $\mathrm{Rb}$ and $\mathrm{p} 53$. Apoptosis. 2008;13(273):81.

13. Cid-Arregui A. Therapeutic vaccines against human papillomavirus and cervical cancer. Open Virol J. 2009; 23(3):67-83.

14. Sterlinko HG, Bergant M, Banks L. Human papillomavirus infection, cancer \& therapy. Indian J Med Res. 2009; 130:277-85.

15. Jalil EM, Duarte G, El Beitune P, Simões RT, Melli PPS, Quintana SM. High prevalence of human papillomavirus infection among Brazilian pregnant women with and without human immunodeficiency virus type 1 . Int J Gynaecol Obstet. 2009;2009:485423. 
16. Cobo F, Concha A, Ortiz M. Human Papillomavirus (HPV) Type distribution in females with abnormal cervical cytology. A correlation with histological study. Open Virol J. 2009;4(3):60-6.

17. Hillemanns P, Breugelmans JG, Gieseking F, Bérnard S, Lamure E, Littlewood KJ, et al. Estimation of the incidence of genital warts and the cost of illness in Germany: a cross-sectional study. BMC Infect Dis. 2008;2(8):76.

18. Villa LL. Prophylactic HPV vaccines: reducing the burden of HPV-related diseases. Vaccine. 2006;30(24):23-8.

19. Giulliano AR, Tortolero-Luna G, Ferrer E, Burchell AN, Sanjose S, Kjaer SK, et al. Epidemiology of human papillomavirus infection in men, cancers other than cervical and benign condictions. Vaccine. 2008;19(26):17-28.

20. Dunne EF, Nielson CM, Stone KM, Markowitz LE, Giuliano AR. Prevalence of HPV infection among men: a systematic review of the literature. J Infect Dis. 2006;15(8):1044-57.

21. Partridge JMA, Koutsky L. Genital human papillomavirus infection in men. Lancet Infect Dis. 2006;6(1):21-31.

22. Giuliano AR, Lazcano- Ponce E, Villa LL, Flores R, Salmeron J, Lee JH, et al. The human papillomavirus infection in men study: human papillomavirus prevalence and type distribution among men residing in Brazil, Mexico and the United States. Salud Pública Mex. 2008;17(8):408-18.

23. Garcia G, Mampaso EG, Someso SC, Pavon EM, Niño SN. Almagro AA. Infección por Papillomavirus en el hombre. Estado Actual. Actas Urol Esp. 2005;29(4):365-72.

24. Rombaldi RL, Serafini EP, Mandelli J, Zimmermann E, Losquiavo KP. Perinatal transmission of human papilomavirus DNA. Virol J. 2009;6:83-94.

25. Campisi G, Giovannelli L. Controversies surrounding human papilloma virus infection, head \& neck vs oral cancer, implications for prophylaxis and treatment. Head Neck Oncol. 2009;30(1):8.

26. Pinto PA, Mellinger BC. HPV in the male patient. Infect Dis Clin North Am. 26(4):797-807.

27. Giuliano AR, Salmon D. The case for a gender-neutral (universal) human papillomavirus vaccination policy in the United States: Point. Cancer Epidemiol Biomarkers Prev. 2008;14(4):805-8.
28. Federação Brasileira das Sociedades de Ginecologia e Obstetrícia-FEBRASGO. Papilomavírus humano(HPV): diagnóstico e tratamento. [acesso em 4 abr. 2010]. Disponível em: http://www.projetodiretrizes.org.br/projeto_diretrizes/079.pdf.

29. Solomon D, Nayar R. Sistema Bethesda para citopatologia cervicovaginal: definições, critérios e notas explicativas. 2a ed. Rio de Janeiro: Revinter; 2005.

30. Rubin MA, Kleter B, Zhou M, Ayala G, Cubilla AL, Quint WGV, et al. Detection and typing of human papillomavirus DNA: evidence for multiple independent pathways of penile. Am J Clin Pathol. 2001;159(4):1211-8.

31. Palefsky J. Human papillomavirus and anal neoplasia. Curr HIV/Aids Rep. 2008;5(2):78-85.

32. Sacramento PR, Babeto E, Cabral MJ, Bonilha JL, Fernandes AM, Pereira JS, et al. The prevalence of human papillomavirus in the oropharynx in healthy individuals in a Brazilian population. J Med Virol. 2006;78(5):614-8.

33. Scheinfeld N, Lehman DS. An evidence-based review of medical and surgical treatments of genital warts. Dermatol Online J. 2006;12(3):5.

34. Tchernev G. Sexually transmitted papillomavirus infections: epidemiology, pathogenesis, clinic, morphology, important differential diagnostic aspects, current diagnostic and treatment options. An Bras Dermatol. 2009;84(4):377-89.

35. Bharti AC, Shukla S, Mahata S, Hedau S, Das BC. Antihuman papillomavirus therapeutics: facts \& future. Indian J Med Res. 2009;130(3):296-310.

36. Yang J, Pu YG, Zeng ZM, Yu ZJ, Huang N, Deng QW. Interferon for the treatment of genital warts: a systematic review. BMC Infect Dis. 2009;21(9):156.

37. Garland SM, Waddell R, Mindel A, Denham IM, McCloskey JC. An open-label phase II pilot study investigating the optimal duration of imiquimod 5\% cream for the treatment of external genital warts in women. Int J Std. AIDS, 2006;17(448):52.

38. Mariani L, Pagliusi S. Vaccination and screening programs: harmonizing prevention strategies for HPV-related diseases. J Exp Clin Cancer Res. 2008;16(27):84.

39. Leon R. Ladies first: should boys be vaccinated against HPV? Can Fam Physician. 2008;54(7):967-8. 
40. Sankaranarayanan R. HPV vaccination: the promise and problems. Indian J Med Res. 2009;130(3):322-6.

41. Bharadwaj M, Hussain S, Nasare V, Das BC. HPV \& HPV vaccination: issues in developing countries. Indian J Med Res. 2009;130(3):327-33.

42. Vera EG, Orozco HH, Soto SS, Aburto EL. Condom effectiveness to prevent sexually transmitted diseases. Ginecol Obstet Mex. 2008;76(2):88-96.

43. Winer RL, Hughes JP, Feng Q, O'Reilly S, Kiviat NB, Holmes KK, et al. Condom use and the risk of genital human papillomavirus infection in young women. $\mathrm{N}$ Engl J Med. 2006;354(25):2645-54.
44. Siegfried N, Muller M, Deeks JJ, Volmink J. Male circumcision for prevention of heterosexual acquisition of HIV in men. Cochrane Database Syst Rev. 2009;15(2):CD003362.

45. Tobian AAR, Serwadda D, Quinn TC, Kigozi G, Gravitt PE, Laeyendecker 0 , et al. Male Circumcision for the Prevention of HSV-2 and HPV Infections and Syphilis. N Engl J Med. 2009;360(13):1298-309.

46. Gray RH, Kigozi G, Serwadda D. Male circumcision for HIV prevention in men in Rakai, Uganda: a randomised trial. Lancet. 2007;369(9562):657-66. 